\title{
Using LaTeX's moodle package and R's Sweave to easily create data-driven, up-to-date financial mathematics and statistics quizzes for Moodle
}

Agnieszka Jach, Department of Finance and Statistics, Hanken School of Economics, Helsinki, Finland. Email: agnieszka.jach@hanken.fi

\begin{abstract}
Preparation of Moodle quizzes which are data-based and contemporary tends to be tedious and time-consuming. By using innovative tools, this process can be simplified and automated, providing a substantial benefit to the teacher wishing to employ such quizzes, and ultimately improving student learning experience. The purpose of this article is to show how to create data-driven, up-to-date quizzes for Moodle in an easy fashion. The methodology is based on several popular, open-source, free tools, and its implementation details are demonstrated with an example. This makes the methodology readily-available to the practitioners.
\end{abstract}

Keywords: reproducible, dynamic, data-dependent, free software, Moodle, R, LaTeX.

\section{Introduction}

Data-driven and up-to-date examples are a good way of engaging the students in the learning process. Likewise, by reflecting the current state of affairs, such examples allow the students to remain informed about the world we live in. Exercises that potentially could benefit from being refreshed regularly are primarily those that depend on short-time-scale data (data observed on daily, monthly, quarterly, possibly annual frequencies), data whose movements follow trends, though arguably some data will remain relevant over longer time scales. In financial mathematics most exercises would lend themselves to being updated periodically as they are concerned with prices of assets (stocks, bonds, exchange rates), often recorded on a daily frequency, prices of derivatives (financial instruments dependent on the underlying assets), risk metrics computed from prices of assets and derivatives, financial statements of companies, credit ratings, levels of interest rates. For example, it is likely that students solving exercisers where interest rates are between $5-10 \%$ per year will find themselves somewhat surprised in view of current economic conditions where interest rates are close to $0 \%$. In statistics, exercises that would benefit from being updated regularly are those that deal with time series data, especially when forecasting is of interest (for example, forecasting monthly electricity production or temperature in the UK for August 2017 in a course given during 2018 might seem somewhat artificial), but also exercises where cross-sectional data collected over time is used.

An implicit step in preparing examples of that kind and employing them for assessment of students' learning is the use of a recent stretch of data, but the time-dependent nature of the examples/exercises means that they cannot easily be re-used - or can they? When the course management system happens to be Moodle (Moodle HQ and Moodle Community (2018)) and the activity that incorporates examples with current data is Moodle's Quiz, then it turns out that the examples can be easily produced and re-produced. One important tool that facilitates data-to-quiz transformation is a recently-developed LaTeX package called moodle (Hendrickson (2016); LaTeX is a type-setting system, Lambert (1994), LaTeX3 Team (2018)). Thanks to this package quiz questions can be embedded into a . tex file and once compiled, the resulting . xml file, containing the quiz questions, can be imported to Moodle. There are several benefits of the moodle package when used as a stand-alone tool, and these are outlined in Hendrickson (2016): a) no web-based 
interface is needed; this feature eliminates the necessity of switching between menus and boxes, making the process of editing questions faster, and it also eliminates the need to rely on the Internet access/speed; b) mathematical and scientific notation can be type-set fast because LaTeX source code is used to produce them, and users have the flexibility of defining their own macros, again to speed up the editing process; c) the . tex file containing quiz questions can be examined, modified, browsed and archived; this is in contrast to editing each question separately in a web-based editor; d) the .pdf file (created at the same time as the . xml file) can be previewed; this allows the user to proof-read the quiz and correct the errors more efficiently compared to the online option. But how does the data-element come into the picture? This step is achieved through R's function Sweave (Leisch (2002), Leisch (2017); R is a computing software, R Core Team (2018)), because Sweave allows one to mix LaTeX syntax and R code (embedded in an . Rnw file) to produce dynamic reports, and of course $\mathrm{R}$ can be used to acquire contemporary data as well as to carry out various analyses.

The goal of this article is to demonstrate how several free, open-source and popular tools can be combined to easily produce data-driven, up-to-date quizzes for Moodle, using examples from economics. Because the exercises involved are produced dynamically, quizzes can be re-cycled, alleviating the problem of time-consuming and painstaking edits, whenever the input data changes.

We give a brief description of each of the above-mentioned tools, though we assume that most of the readers will be familiar with Moodle, $\mathrm{R}$ and LaTeX.

- Moodle and Quiz (activity on Moodle): Moodle is a learning management system employed by many institutions world-wide. One of Moodle's activities is a quiz (Quiz). This module allows one to build quizzes made up of a variety of questions: True/False, multiplechoice, short-answer, matching, numerical, embedded-answers. The last type, termed Cloze in Moodle, consists of a passage of text that has various answers (pertaining to question types just mentioned) embedded within it, and due to its flexibility is a very useful category;

- LaTeX: is a document-preparation system for high-quality typesetting, which allows one to produce professionally-looking mathematical expressions, which can be typed fast using plain text;

- moodle (package for LaTeX): moodle is a package that offers more efficient implementation of Moodle's Quiz compared to the web-based editor. All question types mentioned in the first point (plus an essay) are supported by this package;

- Sweave (function in R): Sweave is used to weave together chunks of R code (so-called code chunks) and chunks of LaTeX syntax (so-called documentation chunks) for reproducible analyses. The source file containing the mixture of both has extension . Rnw. The value of an $\mathrm{R}$ expression, expr, from the code chunk can be used in the documentation chunk via the command \Sexpr\{expr\};

- $R$ and RStudio: $R$ is a programming language and software for statistical computing and graphics and RStudio (RStudio Team (2018)) is an integrated development environment for R.

The rest of the article is organized as follows. In Section 2 we describe the methodology, in Section 3 we give an example of its implementation, finally we provide a brief summary in Section 4 . Supplementary materials (available from the author on request) contain input and output files used in the preparation of this article. 


\section{Methodology}

The pre-requisites for executing the code of this article are: $\mathrm{R}$ including the Sweave function (and RStudio, for convenience); LaTeX compiler with the moodle package installed (distributions for various platforms, for example, Windows's MiKTeX, come with moodle). These programmes are available across different operating systems. If additional $\mathrm{R}$ and LaTeX packages are used (for example, R's Quandle or foptions) by the code and documentation chunks, these are assumed to be installed as well.

The work-flow in preparing the quiz consists of the following three steps. Step 1) In R, create an . Rnw file according to the Sweave guidelines, where the code chunks collect the data and perform analyses and where the documentation chunks elaborate quiz questions with the commands of moodle, as well as making use of values from the code chunks via Sexpr. Step 2) In R, run the . Rnw file using the Sweave function to obtain . tex, .xml and . pdf files. This can be done using RStudio's menus or by creating an R script, which sources the . Rnw file (we present the 2nd option for a potential automation purpose). Step 3) Import the .xml file produced in the previous step to Moodle's Question bank (all quiz questions are uploaded in this single step and are ready to be used for a creation of Quiz activity on Moodle).

Students attempting the quiz presented henceforth (Section 3) are assumed to come from Financetype programmes at a BSc level and are assumed to have knowledge of R (and RStudio) and to be familiar with packages and routines used in financial mathematics and statistics, for example, such as those described in Stander and Eales (2011). The learning objectives associated with the quiz are: students are able to download data sets in $\mathrm{R}$, transform the data, obtain numerical summaries and perform analyses. Such quizzes could form part of formative or summative assessment.

\section{Example}

We give an example of a quiz with three questions (file example.Rnw available in the Supplementary materials). In Question 1 (question type: matching) students are supposed to download two time series in $\mathrm{R}$, transform them, find some numerical summaries of the latter and finally, match the type of a numerical summary with its value. In Question 2 (question type: embedded-answers with numerical) students use the data from Question 1, to find a rational price of a financial instrument called a European option. The price is given via the so-called Black-Scholes formula (well-known in finance), which is implemented in one of R's packages. Here, students have to fill-in-the gaps using numerical values used in the context of the exercise. In Question 3 (question type: embedded-answers with numerical and multiple-choice) students perform Principal Component Analysis on the transformed data from Question 1. Students have to fill-in-the gaps with numerical values or choose one of the options provided from the drop-down menus.

We begin (Step 1) with the file example.Rnw, which we save on our machine, remembering the path to the destination folder. We open example.Rnw in RStudio to view its contents (and optionally, to execute it using RStudio's menus). To carry out Step 2), we save runexample.R (available in the Supplementary materials and shown below) in the same destination folder as example.Rnw, open it in RStudio and complete the line that specifies the working directory. We then execute the code, evoking function Sweave on example.Rnw, sending the output to example.tex. When the last file is compiled, example.pdf and example-moodle.xml are created. What remains to be done is to import example-moodle.xml to Moodle's Question bank and to make the quiz (Step 3). 
\#' runexample. R'

library(tools)

\#specify the working directory (no slash at the end)

mywd<- "C: /Users/..."

setwd (mywd)

myfile<-paste@(mywd, "/example")

Sweave(file=paste@(myfile,".Rnw"), output=paste@(myfile,".tex") )

texi2pdf (file=pasteø (myfile, ". tex"))

One possible extension of the above scheme is to create many variants of the same quiz by changing the input data slightly, for example, by shifting the start and the end dates of the data samples. This can be achieved by embedding the last two lines of runexample. $R$ into a for loop, say for (counter in 1:5), modifying the lines accordingly (output in Sweave and file in texi2pdf), and then using counter to alter the selected lines of example. Rnw appropriately.

To conclude this section we mention several aspects of using the software. One of the known limitations of the moodle package is the lack of shuffling of answers in multiple-choice questions when used inside Cloze question (Section 6 of Hendrickson (2016)), but that feature works correctly when multiple-choice is used as a stand-alone question. The combination of multiple-choice and Cloze questions was employed in Question 3 of the quiz example of this section. One can easily correct for this by editing Question 3 in the web-based Moodle interface. Also some users of moodle might find it difficult to incorporate images into the . $x m l$ file (Section 5 of Hendrickson (2016)). Again, this can be adjusted for by inserting the image via the web-based interface of Moodle. There might be some additional effort required when employing LaTeX's verbatim environment in moodle (potentially-useful environment for code-listings). It is worth pointing out that the moodle package can be used without the involvement of R or RStudio (and Sweave), by simply creating a . tex document with quiz questions and then by compiling it (see also points a)to d) in the introduction of this article), but this does not allow for data retrieval/analysis and creation of dynamic reports. Finally, access to some of the data sets freely-available through R's package Quandl, require the so-called API key. This key can be obtained at no charge from www.quandl.com upon registration at the website.

To finish off, we provide the information about the software type and versions used in the preparation of this article: Operating System: Windows 7 Enterprise; Moodle version 3.3; LaTeX: MiKTeX version 2.9; moodle version 0.5; R: Microsoft $\mathrm{R}$ Open version 3.3.2, RStudio version 1.1.383, Sweave: from R's package utils version 3.3.2. Mac users may wish to consult French (2013), when setting up Sweave.

\section{Summary}

In this article we showed how to create a quiz for Moodle in such a way that it is based on data downloaded and transformed in $\mathrm{R}$. The results of computations and analyses are incorporated into a . tex document using Sweave. Additionally, the . tex document sets quiz questions by employing the LaTeX package moodle. The possibility of automation of the process through exploiting R's capabilities such as loops, allows the user to create different variants of the same question/quiz. This 
could be useful in formative assessments, where students repeat a similar exercise, or in summative assessments, where different students solve different exercises, to ensure academic integrity. We hope that the methodology of this article will benefit practitioners who would like to implement similar quizzes for their courses.

\section{References}

French, J. A., 2013. Easy Sweave for LaTeX and R. Available at: http://www.jasonfrench.com/blog/2013/08/16/easy-sweaving-for-latex-and-r/ [Accessed 20 July 2018].

Hendrickson, A. O. F., 2016. The moodle package: generating Moodle quizzes via LaTeX. Available at: https://ctan.org/tex-archive/macros/latex/contrib/moodle?lang=en [Accessed 20 July 2018].

Lambert, L., 1994. LaTeX: A Document Preparation System. Reading, MA: Addison-Wesley.

LaTeX3 Team, 2018. LaTeX. https://www.latex-project.org/.

Leisch, F., 2002. Sweave: Dynamic generation of statistical reports using literate data analysis. In W. Härdle and B. Rönz, eds. Compstat 2002: Proceedings in Computational Statistics. Heidelberg: Physica Verlag. pp. 575-580.

Leisch, F., 2017. Sweave User Manual. [Available at: https://stat.ethz.ch/Rmanual/Rdevel/library/utils/doc/Sweave.pdf. [Accessed 20 July 2018].

Moodle HQ and Moodle Community, 2018. Moodle: Modular Object-Oriented Dynamic Learning Environment. Moodle project. https://moodle.org/.

R Core Team, 2018. R: A Language and Environment for Statistical Computing. Vienna, Austria: $\mathrm{R}$ Foundation for Statistical Computing. http://www.R-project.org/.

RStudio Team, 2018. RStudio: Integrated Development for R. Boston, MA. http://www.rstudio.com/. Stander, J. and Eales, J., 2011. Using R for teaching financial mathematics and statistics. MSOR Connections, 11(1), pp.7-11. 